\title{
Structural basis for the specific binding between metal ion and chemically modified mismatched base pairs
}

\author{
K. Hirabayashi ${ }^{1}$, S. Adachi ${ }^{1}$, A. Ono ${ }^{2}$, J. Kondo ${ }^{3}$, H. Torigoe ${ }^{1}$ \\ ${ }^{1}$ Department of Applied Chemistry, Faculty of Science, Tokyo University of Science, Tokyo 162-8601, Japan, \\ ${ }^{2}$ Department of Material \& Life Chemistry, Faculty of Engineering, Kanagawa University, Yokohama 221-8686, Japan, \\ ${ }^{3}$ Department of Materials and Life Sciences, Faculty of Science and Technology, Sophia University, Tokyo 102-8554, Japan
}

hirabayashi@rs.tus.ac.jp

The interactions between metal ions and nucleic acids have attracted considerable interest for their involvement in structure formation and folding of nucleic acids, and their possible roles in catalytic activity of nucleic acids. The structural and thermodynamic properties of the binding with the perfectly matched duplex DNA have been reported for many metal ions, but few studies have been reported for the interaction of metal ions with the mismatched base pair duplex DNA. We found that the addition of $\mathrm{Hg}^{2+}$ significantly increased the thermal stability of the duplex DNA with the T:T mismatched base pair [1,2], and the combination of $\mathrm{Hg}^{2+}$ and the duplex DNA with the T:T mismatched base pair was highly specific for metal ion-DNA interactions. Isothermal titration calorimetry (ITC) demonstrated that $\mathrm{Hg}^{2+}$ specifically bound to the $\mathrm{T}$ : T mismatched base pair at 1:1 molar ratio with a binding constant of $10^{6} \mathrm{M}^{-1}$ [2]. We also found that the addition of $\mathrm{Ag}^{+}$significantly increased the thermal stability of the duplex DNA with the C:C mismatched base pair [3, 4], and the combination of $\mathrm{Ag}^{+}$and the duplex DNA with the $\mathrm{C}: \mathrm{C}$ mismatched base pair was highly specific for metal ionDNA interaction. ITC demonstrated that $\mathrm{Ag}^{+}$specifically bound to the $\mathrm{C}: \mathrm{C}$ mismatched base pair at 1:1 molar ratio with a binding constant of $10^{6} \mathrm{M}^{-1}$ [4]. Recently, we analyzed the possibility of specific binding between metal ion and 5-fluorouracil (5-FdU)modified mismatched base pair in duplex DNA. Thermal stability analyses revealed that $\mathrm{Hg}^{2+}$ and $\mathrm{Ag}^{+}$could specifically bind to the T:5-FdU and the C:5-FdU mismatched base pair in duplex DNA, respectively. Here, we determined the crystal structures of two duplex DNAs including mercury-mediated T-Hg- $(5 \mathrm{~F}-\mathrm{dU})$ base pair and silver-mediated C-Ag- $(5 \mathrm{~F}-\mathrm{dU})$ base pair.

Palindromic DNA fragments designed to contain T or C:5-FdU mismatched base pair in the center were used for crystallization. Both fragments were cocrystallized with metal ions by the sitting drop vapor diffusion method, and $\mathrm{x}$-ray diffraction data were collected at the Beamline BL-5A of the Photon Factory. Experimental phases of duplex DNAs containing mercury- and silver-mediated base pairs were obtained by single wavelength anomalous dispersion (SAD) phasing with mercury atoms and the molecular replacement method, respectively. The structure of duplex DNA containing mercury-mediated T-Hg- $(5 \mathrm{~F}-\mathrm{dU})$ base pair was determined at $2.85 \AA$ resolution and revealed that two duplex DNA molecules present in an asymmetric unit. $\mathrm{Hg}^{2+}$ specifically bound to the T:5-FdU mismatched base pair at 1:1 ratio with taking linear coordination to $\mathrm{N} 3$ atoms of $\mathrm{T}$ and 5 -FdU residues to form $\mathrm{T}-\mathrm{Hg}-(5 \mathrm{~F}-\mathrm{dU})$ base pair. The structure of duplex DNA containing silver-mediated C-Ag- $(5 \mathrm{~F}-\mathrm{dU})$ base pair was determined at $2.20 \AA$ resolution and revealed that a singlestranded DNA present in an asymmetric unit; two single-stranded DNAs forming a duplex DNA in the crystal is related by crystallographic 2-fold axis. One $\mathrm{Ag}^{+}$is specifically inserted between C:5-FdU mismatches to form a C-Ag-(5F-dU) base pair by taking linear coordination to $\mathrm{N} 3$ atoms of $\mathrm{C}$ and 5-FdU residues as same as $\mathrm{Hg}^{2+}$. In addition, a hydrogen bond is formed between $\mathrm{O} 4$ atom of 5-FdU and $\mathrm{N} 4$ atoms of $\mathrm{C}$ residue, implying a contribution to stability by forming a stronger bond network.

In this study, we demonstrated the structural basis for the novel interaction of metal ions with the mismatched base pair including 5FdU. Structural information for specific binding modes between chemically modified mismatched base pairs and metal ions can be expected to be applied to various fields such as environment, medicine and nanotechnology.

[1] Ono, A. \& Togashi, H. (2004). Angew. Chem. Int. Ed. 43, 4300-4302.

[2] Torigoe, H., Ono, A. \& Kozasa, T. (2010). Chem. Eur. J. 16, 13218-13225.

[3] Ono, A., Cao, S., Togashi, H., Tashiro, M., Fujimoto, T., Machinami, T., Oda, S., Miyake, Y., Okamoto, I. \& Tanaka, Y. (2008) Chem. Commun. 4825-4827.

[4] Torigoe, H., Okamoto, I., Dairaku, T., Tanaka, Y., Ono, A. \& Kozasa, T. (2012) Biochimie 94, 2431-2440.

Keywords: mismatched base pairs; metal ion; nucleic acid 